\title{
OTIOTOMICS
}

Revista de economía, empresa y sociedad

Trabajo final de máster

CAMINAR HACIA LA IGUALDAD EFECTIVA

\section{Reflexiones sobre la última reforma española de los permisos parentales}

\author{
Alba Fdo Joué \\ Ingeniera de Caminos (UPC). Máster en Dirección y Gestión de RR. HH. (UOC).
}

RESUMEN Cuando el Real Decreto-ley 6/2019 de 1 de marzo, que incorpora, entre otros, la nueva configuración de los permisos parentales, se encuentre totalmente desplegado, España habrá obtenido, en referencia a dichos permisos, la igualdad formal entre hombres y mujeres. Partiendo de una caracterización de la realidad del país respecto a los sesgos de género en el ámbito laboral y doméstico y con el apoyo de la literatura especializada, este artículo argumenta por qué la reforma de los permisos, si bien ha de contribuir en el camino hacia la igualdad efectiva, no promete ser suficiente para mejorar de manera notoria la situación actual. Desde esta información también se propone el sentido en el que debería ser concebida una nueva reforma y se reflexiona acerca de si un país como España podría asumir su coste. Finalmente, se aborda la necesidad de una doble implicación del Estado-tejido empresarial con tal de obtener el cambio disruptivo necesario, no solo en términos de calidad democrática, sino también de ventaja competitiva en el nuevo paradigma socioeconómico.

Este artículo es el resumen de uno de los temas tratados en el trabajo que obtuvo el premio al mejor Trabajo Final de Máster (TFM) de Dirección y Gestión de Recursos Humanos de la UOC, así como el mejor TFM con perspectiva de género en la convocatoria de los premios de Trabajos Finales de los Estudios de Economía y Empresa de la UOC del curso 2018-2019. EI TFM completo, bajo el título de "Las políticas de permisos parentales como elemento clave para romper el techo de cristal y la brecha salarial", está en depósito en el repositorio institucional de la UOC (O2), el portal que recoge, difunde y preserva las publicaciones digitales de libre acceso de los miembros de la UOC, elaboradas en el desarrollo de sus actividades de investigación, docencia y gestión. Se puede consultar en este enlace: http://hdl.handle.net/10609/100467.

PALABRAS CLAVE igualdad de género; mercado laboral; penalización maternidad; permisos parentales 


\title{
Reflections on the latest Spanish reform of parental leave
}

\begin{abstract}
Once the Royal Decree-Law 6/2019 of 1 March, incorporating and including the new configuration for parental leave, has been completely rolled out, Spain will have obtained formal equality between men and women with respect to said leave. Starting from a description of reality in the country regarding gender biases in the labour and domestic spheres and with the support of the specialised literature, this article argues why the reform of parental leave, although it should make a contribution in the path towards effective equality, does not look to be enough for improving the current situation in a significant way. Working from this information, the way in which a new reform should be conceived is also proposed, and there is a reflection on whether a country such as Spain could pay for its cost. Finally, there is an analysis of the need for a double involvement of the state and the business world, along with the need for obtaining the requisite disruptive change, not only in terms of democratic qualities but also in terms of competitive advantage in the new socioeconomic paradigm.
\end{abstract}

This article is the summary of the work which obtained the prize for the best Master's Dissertation (TFM) in Human Resources Management at the UOC, as well as the best dissertation concerning gender studies in the competition for the Master's Dissertation prizes in Economics and Business Studies at the UOC during the 2018-2019 course. The complete TFM, under the title "Parental leave policies as a key element for breaking the glass ceiling and ending the wage gap", is held in the UOC's institutional repository (O2), the portal which collects, disseminates and preserves the UOC members' free-access digital publications that were produced in the development of their research, teaching and management activities. It can be viewed at this link: http://hdl.handle.net/10609/100467.

KEYWORDS gender equality; labour market; motherhood penalty; parental leave

\section{Introducción}

A medida que las sociedades caminan hacia la igualdad de género, también de la mano de las ordenanzas jurídicas, la discriminación histórica que ha otorgado al hombre un rol predominante como proveedor por medio del trabajo remunerado en el espacio público y ha reservado a las mujeres el trabajo no remunerado en el ámbito doméstico y privado, ha ido menguando en favor de la disolución de los roles de género y sus consecuencias dentro y fuera de los hogares.

A pesar de todo, existe aún en el imaginario colectivo, consciente o inconsciente, la idea de que la responsabilidad del trabajo reproductivo es de las mujeres. La norma no escrita que impera en el seno de las familias resta tiempo a las mujeres para acceder al espacio público y libera a los hombres para seguir cultivando sus oportunidades en el mercado del trabajo remunerado gracias a las tareas domésticas realizadas por ellas (Guzmán, 2003). Así, cuando estas han querido o han podido incorporarse al mercado laboral se les ha impuesto una doble jornada que les ha impedido desarrollarse profesionalmente en igualdad de condiciones. Esta realidad tiene una transposición directa en la brecha salarial de género, pero también en el grado de responsabilidad que las mujeres tienen la oportunidad de asumir en las organizaciones, el llamado techo de cristal. Su efecto se ve magnificado por la maternidad, ya sea directamente o por discriminación estadística.

Las reflexiones que aquí os presentamos parten del convencimiento de que en una sociedad donde formalmente se vela por eliminar la discriminación de género en el trabajo, existe una realidad social que condiciona las 
oportunidades y la manera en que la mujer participa en el mercado laboral. Si nos enmarcamos en el contexto actual de la reforma española de los permisos por nacimiento de hijos que se incluye en el relativamente reciente Real Decreto-ley 6/2019 de 1 de marzo, es prácticamente obligado cuestionarse la idoneidad y suficiencia de las medidas que la nueva ley implementa con el fin de obtener o fomentar la igualdad de género tanto en la esfera profesional como en la doméstica.

\section{Caracterización de la realidad española. Sesgos de género en el mercado laboral y en los usos del tiempo}

Los distintos indicadores del INE permiten concluir que la segregación de roles tiene una marcada presencia tanto en la esfera doméstica como en la profesional y que, a pesar de que la incorporación de la mujer en el mercado laboral es un hecho desde hace años, la economía familiar española se basa en el modelo de "gana pan y medio» (término introducido en Castro, 2015).

Algunos valores ejemplifican esta afirmación. Mientras que un escaso 6,84 \% de los hombres trabaja a tiempo parcial, el porcentaje crece hasta el $24 \%$ para las mujeres. Si bien es cierto que el motivo principal para ambos géneros es el de no haber encontrado un trabajo a jornada completa, en la población entre 25 y 64 años, el porcentaje de mujeres que aluden a motivos relacionados con el trabajo reproductivo no remunerado supera el 22 $\%$, mientras que no llega al $4 \%$ para los hombres. El techo de cristal se hace patente con el escaso 30,6 \% que representa la presencia de las mujeres en los cargos de mayor responsabilidad de las empresas y la brecha salarial en referencia al salario bruto, que ronda el 21,9 \% (INE, 2017). Si bien es cierto que esta cifra disminuye hasta el $13,5 \%$ si se tiene en cuenta el salario por hora (INE, 2017), al considerar la «brecha de ingresos» (indicador síntesis proporcionado por Eurostat que mide el impacto de la combinación de tres factores: media del salario por hora; media de horas mensuales pagadas -asimilable al tipo de jornada-, y tasa de ocupación) el valor se eleva, para España, hasta el 35,7 \% (última actualización de datos: 2014). A pesar de todo, el dato más esclarecedor de la realidad de la división sexual del trabajo es el que involucra a la esfera doméstica, donde las mujeres dedican en torno a 4,5 horas diarias a las tareas del hogar y el cuidado de la familia, casi el doble de la dedicación de los hombres y se constata que el desequilibrio no responde a la incidencia del tipo de jornada (Edo, 2019).

\section{La penalización de la maternidad frente al premio a la paternidad}

En España, como en el resto del mundo, los efectos de la maternidad/paternidad en el mercado laboral suponen una dualidad entre penalización y recompensa. Dos factores son determinantes en la penalización de la maternidad que paralela e intrínsecamente dejan espacio para premiar la paternidad.

El primero es la priorización -voluntaria o por necesidad- por parte de las mujeres de la compatibilidad del trabajo con las responsabilidades familiares, lo que provoca una pérdida de flexibilidad.

El segundo es la integración por parte de los empleadores de esta priorización. La organización social afecta a la lógica empresarial que, al definir su estrategia adaptativa a la cultura y a la sociedad en cuanto a los recursos humanos, fomenta la discriminación en términos de contratación, de oportunidades profesionales y, consecuentemente, de estructura salarial, hecho que al mismo tiempo determina el poder de negociación y las decisiones personales.

La maternidad tiene un efecto claro en el aumento de la brecha salarial y la densificación del techo de cristal (Dueñas y Moreno, 2017; López, et al., 2018; Cebrián y Moreno, 2008; Castro, 2015). Es más, según apuntan algunos estudios, la maternidad reduce la empleabilidad y los ingresos de las madres respecto de las que no lo son hasta los 44 años, mientras que para los hombres tener hijos supone una mejor situación en el mercado laboral a partir de los 33 años (López, et al., 2018), es decir, que los premia. A partir de los 44 años, las mujeres con hijos 
recuperan terreno respecto a las que los tienen. Es la edad en la que las madres regresan al sector privado y a las jornadas a tiempo completo (Dueñas y Moreno, 2017), abandonando el sector público y la jornada parcial que les ha servido de refugio laboral durante los años de crianza de los hijos pequeños. Este fenómeno, ligado a las ventajas fiscales por descendencia que se aplican en el estado español, podría explicar la inversión de la tendencia.

Es en este sentido que es necesario considerar la capacidad transformadora de las políticas públicas, y en concreto las referentes a las de los permisos parentales, pues pueden marcar la diferencia entre la perpetuación de la segregación de roles y la promoción del cambio disruptivo que hará caminar la sociedad hacia la igualdad efectiva.

\section{El permiso parental con la ley vigente}

Con el objetivo de dotar a la sociedad española de un marco jurídico enfocado en la obtención de la igualdad efectiva, el Real Decreto-ley de 1 de marzo, modificaba la Ley Orgánica 2/2007 de 22 de marzo, estableciendo, entre otros, los siguientes cambios en el Estatuto de los Trabajadores: ${ }^{1}$

- El permiso por nacimiento, adopción, guarda con finalidad de adopción o acogida es de 16 semanas por cada uno de los progenitores. Las primeras 6 semanas después del nacimiento son obligatorias para ambos. Es un derecho individual intransferible. Hasta la entrada en vigor de la ley, las 6 semanas solo eran obligatorias para la madre biológica y el resto del periodo era transferible, considerando que, en el caso de la adopción, las 16 semanas eran a repartir entre los dos progenitores -art. 48.4 y $48.5-$.

- El permiso por lactancia (diario o acumulado) es un derecho individual y puede ser ejercido por ambos progenitores. Hasta la entrada en vigor de la ley, solo podía disfrutarlo uno de los dos -art. 37.4-.

- Se añade la disposición transitoria decimotercera que fija el calendario de aplicación en tres fases: 01/04/2019, 01/01/2020 y 01/01/2021.

Las excedencias por cuidado de los hijos/as no se ven modificadas. Se trata de un derecho individual, no remunerado, con una duración máxima de tres años a contar desde el nacimiento o la resolución de adopción (art. 46.3 ET).

Tampoco varían las condiciones del derecho a la reducción de jornada por guarda de menores que sigue siendo un derecho individual que permite reducir la jornada, con reducción proporcional del salario, entre un mínimo de una octava parte y un máximo de la mitad de la jornada laboral, hasta que el menor tiene 12 años (art. 37.6 ET).

\section{El uso del permiso por parte de los hombres}

Las condiciones y consecuencias del uso del permiso por parte de los hombres son dos puntos clave a la hora de analizar la idoneidad de las medidas de la nueva ley.

En primer lugar, es necesario considerar que el uso del permiso por parte de los hombres contribuye a una implicación superior que permanece en el tiempo respecto al cuidado de los hijos (Meil, 2011; Castellanos et al., 2013) y que esta, a su vez, diluye los roles de género en las familias biparentales (Castro, 2015; Castro y Pazos, 2012). En este sentido es interesante la aportación de Castro (2015) en la que evidencia la potencialidad génerotransformativa de las políticas de los permisos en la disolución de la segregación de roles.

1. En términos de permisos por nacimiento, adopción y lactancia los cambios también son aplicables al personal laboral en servicio de las administraciones públicas, cuyas relaciones laborales se encuentran reguladas por la Ley del Estatuto Básico del Trabajador Público. 
Estos estudios refuerzan la idea de que la igualdad y la disolución de los roles de género pasan por un cambio cultural que puede relacionarse con el uso del permiso por parte de los padres.

En según lugar, pues, es necesario determinar cuáles son las condiciones en las que los hombres hacen uso de este permiso.

En el contexto español, conforme ha ido aumentando la parte intransferible remunerada, los padres han respondido a la medida usando dicho periodo exclusivo remunerado hasta mantenerse en una proporción de un 84 $\%$ de los hombres que usan el permiso (Castro, 2015).

No ha sucedido lo mismo con la parte que hasta el Real Decreto-ley 6/2019 era transferible -con un porcentaje de uso de un 2 \% por parte de los hombres (Lapuerta, 2011; Lapuerta, 2012)-. En algunas comunidades autónomas se ha intentado incentivar con una pequeña remuneración extra, pero no ha resultado efectivo (Castro, 2015).

Si nos fijamos en las solicitudes del permiso de excedencia por cuidado de hijos menores de tres años, que en España es intransferible y no remunerado, solo el 7,66 \% se realiza por hombres (Instituto de la Mujer, 2018)2.

Estos datos ponen sobre la mesa los dos ejes de decisión que condicionan el uso del permiso por parte de los padres y de los que conjuntamente determinan el paso de la igualdad formal a la efectiva: la intransferibilidad y la remuneración. De igual manera, evidencias empíricas halladas por distintos estudios como los de Castro y Pazos (2007, apoyadas en Ekberg et al., 2003 y Eriksson, 2005), Lapuerta (2012) y Castro (2015) concluyen que la intransferibilidad de los permisos hace que los padres usen su cuota siempre y cuando esta cumpla las expectativas de remuneración determinadas por los ingresos previos, es decir, que el permiso no solo ha de estar remunerado, sino que tiene que considerar un alto nivel de cobertura del salario.

Por su lado, el Banco Mundial reconoce que las políticas de permisos parentales que consideran a ambos progenitores y lo hacen de manera preceptiva (como Islandia, Noruega o Suecia), presentan la ventaja de reducir la penalización (directa o estadística) que supone la maternidad ante lo que ocurre con aquellas políticas que solo consideran a las mujeres. Según el organismo, este fenómeno se refleja en dos sentidos: reduciendo las decisiones discriminatorias de los empleadores y desplazando las normas subyacentes de la segregación de roles respecto a los cuidados familiares (World Bank, 2012). En concreto, los permisos individuales no transferibles reducen el poder de negociación potencialmente asimétrico dentro de la familia, las presiones laborales y el coste de transgredir los roles de género tradicionales (Castellanos, 2016).

\section{Idoneidad y suficiencia de las nuevas medidas}

Si consideramos la dinámica de los hombres españoles de agotar el tiempo exclusivo de permiso siempre y cuando este sea remunerado, es plausible aceptar que, al aumentar el tiempo hasta las 16 semanas, la igualdad formal en los permisos remunerados se reflejará en la realidad de su uso. Por lo tanto, en consideración a la potencia género-transformativa del uso del permiso por parte de los hombres vista anteriormente, la nueva ley tendría que contribuir a diluir el marcado sesgo de género en la esfera doméstica y este efecto tendría que trasponerse al ámbito laboral.

Ahora bien, no podemos olvidar que las nuevas medidas no plantean ningún cambio en referencia al permiso de cuidado de los hijos menores de 3 años. Este seguirá siendo no remunerado y no habrá ningún aliciente para aumentar el porcentaje de hombres que lo soliciten.

Es decir, que, resultados en mano, es esperable cierta mejora en el reparto de los usos del tiempo a nivel doméstico. Pero será difícil el cambio disruptivo en la mentalidad de los empleadores y, en definitiva, en la empleabilidad y los condicionantes de participación en el mercado laboral de las mujeres respecto de los hombres. De este modo, será complicado romper la retroalimentación que hoy en día aún perdura entre el reparto desigual del

2. http://www.empleo.gob.es/es/estadisticas/contenidos/anuario.htm. 
trabajo doméstico no remunerado, las oportunidades y los recursos disponibles de las mujeres para desarrollar su carrera profesional y el posicionamiento que los empleadores permiten de estas.

Así pues, se reconoce el paso adelante que supondrá la nueva ley, una pequeña victoria más en la lucha por la obtención de la igualdad efectiva, pero el camino por recorrer es todavía largo y no exento de dificultades.

\section{El coste de caminar hacia la igualdad efectiva. El beneficio de la disolución de roles}

Por tanto, parece que existe una barrera en la configuración de los permisos que, contextualizada aún en una imperante segregación de roles de género, impide una verdadera promoción de la participación de los hombres en el cuidado de los hijos. Si añadimos la relación entre este involucramiento de los hombres y la disminución de los sesgos de género en el trabajo doméstico no remunerado, y recuperamos la premisa de la disminución de la empleabilidad -o directamente la expulsión involuntaria de la población activa-, que representa para las mujeres la imposición de la doble presencia, resulta evidente que, hasta las políticas más avanzadas de permisos parentales carecen de una estructuración género-transformativa que tenga como objetivo acabar con la división sexual del trabajo. Esta debería basarse en una composición que considerase evidente la simetría de género en los elementos normativos, pero además de los aspectos formales, tendría que buscar la implicación activa de los hombres mediante permisos intransferibles y bien remunerados, de manera que se abandonara el enfoque maternalista que aún impera en la cultura.

Esto nos lleva a pensar que, en el estado español, más allá de la implementación de la nueva ley que reforma la concepción de los permisos de maternidad y paternidad, hace falta plantearse la necesidad de un cambio que también varíe la configuración actual de la excedencia por cuidado de los hijos, ya que esta, si bien reserva el lugar de trabajo de quien la utiliza, sigue contribuyendo a perpetuar la segregación tradicional de los roles de género.

En este sentido, aún resulta más evidente la necesidad de cuestionarse qué implicaciones económicas tienen estas reformas. ¿Puede un país como España asumir el coste? Este es en cualquier caso un tema para abordar con mayor profundidad, pero las preguntas no pueden desligarse de otras cuestiones igualmente necesarias. ¿Puede una sociedad que aspira a ser competitiva permitirse el coste de continuar perpetuando una fuente de discriminación como es la división sexual del trabajo?

En términos de coste económico, un primer orden de magnitud nos lo da el estudio publicado en la web de la organización «Iguales e intransferibles» ${ }^{3}$ que cifra el coste del aumento de las 5 semanas de permiso de paternidad a las 16 en un incremento del gasto anual de unos 1.100 millones de euros. Sin analizar otras inversiones estatales de mayor valor y dudoso beneficio social, vale la pena tener presente el dato proporcionado por Medialdea y Pazos (2011), quienes estimaban en más de 2.600 millones de euros las pérdidas de aquel año en la recaudación del IRPF bajo la modalidad «declaración conjunta» - una posibilidad que a la vez promociona la permanencia de las mujeres fuera del mercado laboral o bien en la economía sumergida.

Un estudio más detallado podría determinar los costes económicos de implementar permisos para cuidado de hijos diseñados para fomentar la verdadera igualdad, pero sería conveniente plantearse una configuración de distinta naturaleza a la actual, con permisos por cuidado de hijos (actual excedencia de hasta 3 años) más cortos pero altamente remunerados, ya que, por otro lado, tampoco se puede menospreciar el peligro que suponen para la continuidad en el mercado laboral la promoción profesional y las ausencias de larga duración. Lo que nos lleva a reflexionar también sobre la conveniencia de mejorar el sistema estatal de preescolarización y/o plantear la necesidad de establecer cierta normativa de uso consecutivo de los permisos por parte de los dos progenitores, un aspecto que al mismo tiempo promovería la corresponsabilidad.

3. https://igualeseintransferibles.org/wp-content/uploads/2018/10/Cifras-coste-beneficio-reforma-PPIINA-actualizado-a-2018.pdf. 
Pero en el estudio coste/beneficio es de capital importancia considerar el hecho de que la promoción de la igualdad no es solo una cuestión de ética o de calidad democrática, sino que supone una oportunidad para aprovechar todo el capital cuidador de los hombres y todo el potencial productivo y de talento de las mujeres sin estereotipos condicionados ni condicionantes.

Esta optimización de recursos, más allá de propiciar una mayor eficiencia en el conjunto de la sociedad, también supone, en el plano concreto, una fuente de ventaja competitiva para las empresas. Es cierto que a priori las reformas exigen un sobresfuerzo por parte de las organizaciones en términos de reemplazo de aquellos hombres que hasta ahora no disfrutaban del permiso de paternidad o de la excedencia por cuidado de hijos. Del mismo modo, las bajas suponen un coste asociado extra que con el ordenamiento jurídico actual no siempre es compensable mediante beneficios fiscales. Pero la dilución de los roles de género tiene beneficios asociados difícilmente rechazables en el nuevo paradigma que representa la era del conocimiento, donde los activos intangibles han aumentado su valor potencial hasta superar con creces la potencialidad de los activos materiales.

Una distribución más equitativa del trabajo remunerado tiene implicaciones directas en el aumento de la empleabilidad de las mujeres. Esta transformación, especialmente contextualizada en una sociedad donde el porcentaje de mujeres en niveles altos de formación superan el de los hombres, supone una ampliación de la oferta a la hora de reclutar y retener talento de forma efectiva. Al tiempo que aleja el reparto de oportunidades internas y externas bajo un criterio de género, lo aproxima a un criterio meritocrático mucho más equitativo y eficiente en términos de gestión de talento. Una gestión del talento que considere la igualdad de oportunidades, a su vez, genera un mayor grado de satisfacción con el trabajo, mejora la autoestima de los colaboradores, su autoconfianza y la relación con los estamentos superiores de la organización -aspectos, todos ellos, que redundan en un mayor compromiso con la empresa- por no olvidar la importancia de la diversidad de género a la hora de contar con una adaptación más eficiente a los mercados constantemente cambiantes.

Por tanto, en términos de implementación de políticas que busquen la igualdad efectiva entre hombres y mujeres, es necesaria una doble implicación Estado-tejido empresarial.

El Estado tiene que destinar por un lado partidas presupuestarias -lo que es factible considerando los costes descritos y sobre todo el beneficio que implica para la sociedad del bienestar-, y por otro lado tiene que facilitar otros recursos a las empresas, por ejemplo, con programas de beneficios fiscales y ayudas, para que la implementación de estas políticas no sea a priori traumática, ya que no podemos perder la perspectiva de que el $95 \%$ del tejido empresarial español son micropymes con unos recursos económicos mucho más sensibles al aumento de los reemplazos temporales que suponen estas medidas.

Por parte de las empresas es evidente que la adaptación supone destinar recursos económicos que, a pesar de que acabarán teniendo beneficios, no siempre serán inmediatos. Pero su implicación también tiene el componente de la responsabilidad social. Sin obviar que la igualdad entre hombres y mujeres se halla entre los ámbitos de actuación prioritaria de la Alianza Europea por la Responsabilidad Social de las Empresas, esta es también una oportunidad para el posicionamiento ante los mercados cada vez más exigentes en referencia al compromiso social que las organizaciones toman con el entorno donde desarrollan su actividad.

Finalmente, hay que tener presente que, en cuestiones de igualdad, y esto también es válido para el resto de colectivos que sufren discriminación, las políticas sociales y el cambio en las culturas organizacionales tienen que ser la punta de lanza que transforme mentalidades y contextos. Las sociedades no se pueden permitir dejar atrás a ninguno de sus integrantes. Todos y cada uno de ellos conforman, individual y colectivamente, una oportunidad de incorporar talento; y el talento, especialmente en el nuevo paradigma donde el conocimiento toma un absoluto protagonismo, es la clave para obtener competitividad, no solo en términos económicos, sino también sociales. Esta es, sin ningún tipo de duda, una condición a la que toda nación tendría que aspirar $y$, sin embargo, es un hito inalcanzable si no se destinan los medios suficientes para obtener una igualdad de género efectiva. 


\section{Bibliografía}

CASTELLANOS, C. (2016). Evaluación de políticas públicas: los regímenes económicos matrimoniales y los sistemas de permisos parentales. Efectos sobre el régimen de bienestar desde la perspectiva de género. Memoria para optar al grado de Doctor. Universidad Complutense de Madrid. Disponible en: https://eprints.ucm. es/37399/.

CASTELLANOS, C. et al. (2013) «¿Se implican más en el cuidado de sus hijos pequeños los padres que utilizaron la baja por nacimiento? El caso de España». Actas del IV Congreso de la Red Española de Política Social (REPS): «Las politicas sociales entre crisis y post-crisis». Pág. 957-977. Disponible en: http://www3.uah.es/ congresoreps2013/.

CASTRO, C. (2015). Modelos de bienestar, igualdad de género y permisos por nacimiento en un contexto de crisis del modelo social europeo. Tesis Doctoral. Departamento de Economía, Métodos Cuantitativos e Historia Económica. Universidad Pablo Olavide. Sevilla.

CASTRO, C.; PAZOS, M. (2007). «Permisos de maternidad, de paternidad y parentales en Europa: algunos elementos para el análisis de la situación actual». Papeles de trabajo. Instituto de Estudios Fiscales (núm. 26). Madrid.

CASTRO, C.; PAZOS, M. (2012). «Permisos por nacimiento e igualdad de género: ¿Cómo diseñar los permisos de maternidad, paternidad y parentales para conseguir un comportamiento corresponsable?». Papeles de trabajo. Instituto de Estudios Fiscales (núm. 9). Madrid.

CEBRIÁN, I.; MORENO, G. (2008). «La situación de las mujeres en el mercado de trabajo español: desajustes y retos». Economía Industrial. Núm. 367, pág. 121-137.

DUEÑAS, D; MORENO, A. (2017). «Mujeres, madres y trabajadoras. Incidencia laboral de la maternidad durante el ciclo económico 2005 - 2016». Revista de Economía Laboral. Vol. 14, núm. 2, pág. 66-103. https://doi. org/10.21114/rel.2017.02.04.

EDO, A. (2019). «Les polítiques de permisos parentals com a element clau per trencar el sostre de vidre i la bretxa salarial». Disponible en: http://hdl.handle.net/10609/100467.

EKBERG, J. et al. (2003): «Sharing Responsibility? Short and Longterm Effects of Sweden's 'Daddy-Month' Reform». WP SOFI [Citado en Castro y Pazos (2007)].

ERIKSSON, R. (2005). Parental Leave in Sweden: The Effects of the Second Daddy Month. Disponible en: http:// swopec.hhs.se/sofiwp/abs/sofiwp2005_009.htm [Citado en Castro y Pazos (2007)].

GONZÁLEZ, M. J.; JURADO, T. (2009). «¿Cuándo se implican los hombres en las tareas domésticas? Un análisis de la Encuesta de Empleo del Tiempo». Panorama Social. Núm. 10, pág. 65-81.

GUZMÁN, V. (2003): «Gobernabilidad democrática y género, una articulación posible». CEPAL: Unidad Mujer y Desarrollo. Serie Mujer y Desarrollo. Núm. 48. Santiago de Chile. Disponible en: https://repositorio.cepal.org/ bitstream/handle/11362/5911/1/S038566_es.pdf.

LAPUERTA, I. (2011). "Individual and Institutional Constraints: An Analysis of Parental Leave Use and Duration in Spain», Population Research and Policy Review. Núm. 30, pág.185-210. https://doi.org/10.1007/s11113010-9185-y.

LAPUERTA, I. (2012). Employment, Motherhood and Parental Leaves in Spain. Tesis Doctoral Universitat Pompeu Fabra.

LÓPEZ, M.T. et al. (2018). «¿Existe penalización por maternidad? Mujeres y mercado laboral en España desde una perspectiva de familia». Estudio realizado por la Unidad de Investigación y Estudios de Familia de la ONG Acción Familiar con la participación de la Universidad Complutense de Madrid y la Universidad de Sevilla. Ediciones Cinca.

MEDIALDEA, B.; PAZOS, M. (2011) «Impuesto sobre la renta español (IRPF) desde la perspectiva de género: cuestiones de equidad y de eficiencia». XVIII Encuentro de economía pública. Disponible en: https://dialnet. unirioja.es/.

MEIL, G. (2011). «El uso de los permisos parentales por los hombres y su implicación en el cuidado de los niños en Europa». Revista Latina de Sociología. Vol. 1, pág. 61-97. 
MEIL, G.; ESCOBEDO, A. (2018). «Gender Equality and Parental Leave». Revista Española de Sociología. 2018 Supplement. Vol. 27, pág. 9-12. https://doi.org/10.22325/fes/res.2018.30.

PAZOS, M. (2010) «Permisos de paternidad: ¿cuotas masculinas o reparto equitativo de los cuidados entre hombres y mujeres?». Publicado en la web de la Plataforma por Permisos Iguales e Intransferibles de Nacimiento y Adopción (PPiiNA). Disponible en: https://igualeseintransferibles.org/blog/permisos-de-paternidad-cuotasmasculinas-o-reparto-equitativo-de-los-cuidados-entre-hombres-y-mujeres/.

PAZOS, M. (2013). Desiguales por Ley. Las políticas públicas contra la desigualdad de género. Libros La Catarata. Disponible en: http://mariapazos.com/wp-content/uploads/2013/11/Desiguales-Por-Ley-PDF.pdf.

WORLD BANK (2012). «World Development Report 2012: Gender Equality and Development. World Bank». Informe del Banco Mundial. Washington DC.

Cita recomendada: EDO, Alba. Caminar hacia la igualdad efectiva. Reflexiones sobre la última reforma española de los permisos parentales. Oikonomics [en línea]. Mayo 2020, no. 13, pp. 1-9. ISSN: 2339-9546. DOI: https://doi.org/10.7238/o.n13.2008

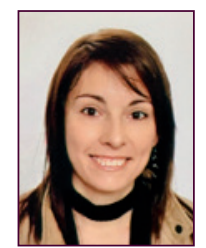

\section{Alba Fdo Jové}

albaej@uoc.com

Ingeniera de Caminos (UPC). Máster en Dirección y Gestión de Recursos Humanos (UOC).

Ingeniera de Caminos por la Universitat Politècnica de Catalunya y Máster en Dirección y Gestión de Recursos Humanos con Premio Extraordinario por la Universitat Oberta de Catalunya. Su trabajo «Las políticas de permisos parentales como elemento clave para romper el techo de cristal y la brecha salarial» obtuvo el premio al mejor Trabajo Final de Máster de Dirección y Gestión de Recursos Humanos, así como el mejor TFM con perspectiva de género en la convocatoria de los premios de Trabajos Finales de los Estudios de Economía y Empresa de la UOC del curso 2018-2019.

Los textos publicados en esta revista están sujetos -si no se indica lo contrario- a una licencia de Reconocimiento 4.0 Internacional de Creative Commons. Puede copiarlos, distribuirlos, comunicarlos públicamente, hacer obras derivadas siempre que reconozca los créditos de las obras (autoría, nombre de la revista, institución editora) de la manera especificada por los autores o por la revista. La licencia completa se puede consultar en https://creativecommons.org/licenses/by/4.0/deed.es_ES.

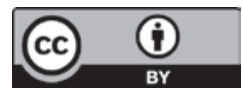

